\title{
IMPLEMENTATION OF THE INVERSE RADON TRANSFORM BY OPTICAL CONVOLUTION
}

\author{
M. NISHIMURA *, D. PSALTIS, F. CAIMI and D. CASASENT \\ Camegie-Mellon University, Department of Electrical Engineering, \\ Pittsburgh, PA 15213, USA
}

Received 28 December 1977

Revised manuscript received 8 March 1978

\begin{abstract}
The implementation of the inverse radon transformed by optical correlation is described. This method described does not require formation of the derivative of the projections and allows the use of a fixed mask that is both real and positive and is dependent only on the geometry of the recording system. Experimental confirmation of the concept is included.
\end{abstract}

\section{Introduction}

The inverse radon transform [1] describes how recorded back projections can be summed to produce an exact reconstruction of the image slice. This is the key operation used in the computer assisted transaxial (CT) X-ray imaging and other systems in which a reconstruction is obtained from projections [2-4]. The form of the reconstruction algorithm we use was formulated by Vest and others [5-7]. The hypersurface involved is discussed by Gel'Fand et al. [7].

In section 2, we describe the CT geometry and notation to be used and the formulation of the inverse radon transform in a form compatible with its optical realization. This derivation differs from our earlier one [8], in that differential operations are not required and a positive reference mask function is used. In section 3, the optical system used is described and our experimental results presented.

\section{Mathematical formulation}

The operation of transaxial imagery construction from projections is well known $[2,4]$. We denote

\footnotetext{
* M. Nishimura is an Associate Professor at Maizuru Technical College, Kyoto, Japan. He was a visiting professor at CMU while this work was performed.
}

the absorption coefficient along a slice $z^{\prime}$ of the input object by $\mu(x, y)$. Polar coordinates are generally used in which case we describe the input object or desired reconstruction by $\mu(r, \theta)$. The collected data from which $\mu(r, \theta)$ is to be obtained are the projections $f$ at different projection angles $\phi$. These projections are recorded along a line $x^{\prime}$ (normalized from +1 to -1 ) normal to the direction of projection. We denote these projections by $f\left(x^{\prime}, \phi\right)$. They consist of the recorded energy absorption along the line $x^{\prime}$ that is normal to specify projection angles $\phi_{\mathrm{m}}$.

The exact reconstruction of $\mu(r, \theta)$ from these projections is given by

$\mu(r, \theta)=\frac{1}{2 \pi^{2}} \int_{0}^{\pi} \mathrm{d} \phi \int_{-\infty}^{\infty} \frac{\partial f\left(x^{\prime}, \phi\right)}{\partial x^{\prime}} \frac{\mathrm{d} x^{\prime}}{r \cos (\phi-\theta)-x^{\prime}}$

The $x^{\prime}$ integral in (1) must be treated as a principal value. By partial integration, (1) can be written with arguments $f\left(x^{\prime}, \phi\right)$ and $h_{1}\left[r \cos (\phi-\theta)-x^{\prime}\right]$, thereby eliminating the derivative in (1). Care must be taken in choosing $h_{1}$ proportional to the derivative of the principal value of $\left[r \cos (\phi-\theta)-x^{\prime}\right]^{-1}$. Several functions satisfying this exist [2]. $h_{1}$ is usually bipolar with the positive part approximating a delta function and the negative part proportional to $\left[r \cos (\phi-\theta)-x^{\prime}\right]^{2}$.

Our present concern is to demonstrate a novel optical implementation of the Radon transform. We thus chose $h_{1}=\left[r \cos (\phi-\theta)-x^{\prime}\right]^{2}$, neglect the delta func- 
tion, and approximated (1) by

$\mu(r, \theta)=-\frac{1}{2 \pi^{2}} \int_{0}^{\pi} \mathrm{d} \phi \int_{-\infty}^{\infty}\left\{\frac{f\left(x^{\prime}, \phi\right)}{\left[r \cos (\phi-\theta)-x^{\prime}\right]^{2}}\right\} \mathrm{d} x^{\prime}$.

In practice the $h_{1}$ function used has a flattened top similar to the $h_{\mathrm{t}}\left(x^{\prime}\right)$ function in eq. (4.30) of [2] truncated to a point $x_{\mathrm{m}}=\epsilon$ as in eq. (4.32) of [2]. Bipolar functions would clearly be superior, but our present purpose is to present and demonstrate a novel optical implementation of the inverse Radon transform. Implementation of the image reconstruction by eq. (2) is preferable to implementation using eq. (1) as we shall show.

We first rearrange eq. (2) in a form more suitable for optical implementation. We interchange the order of integration. This is allowed since the functions $f$ and $\left[r \cos \phi-x^{\prime}\right]^{-2}=g\left(r, x^{\prime}, \phi\right)$ are bounded. We then recognize that

$$
\int \frac{f\left(x^{\prime}, \phi\right)}{\left[r \cos (\phi-\theta)-x^{\prime}\right]^{2}}=f\left(x^{\prime}, \phi\right) * \frac{1}{\left[r \cos \phi-x^{\prime}\right]^{2}},
$$

where ${ }^{*}$ denotes convolution over $\phi$.

$\mu(r, \theta)=-\frac{1}{2 \pi^{2}} \int_{-\infty}^{\infty} \mathcal{F}_{\nu}^{-1}\left[F_{\phi}\left(x^{\prime}, \nu\right) G_{\phi}\left(r, x^{\prime}, \nu\right)\right] \mathrm{d} x^{\prime}$,

where $\mathcal{F}$ denotes the Fourier transform, $\mathscr{F}^{-1}$ denotes the inverse Fourier transform, $F_{\phi}\left(x^{\prime}, \nu\right)$ is the one dimensional Fourier transform in $\phi$ of $f\left(x^{\prime}, \phi\right)$, $G_{\phi}\left(r, x^{\prime}, \nu\right)$ is the one dimensional Fourier transform in $\phi$ of $g\left(r, x^{\prime}, \phi\right)$ and use has been made of the fact that the convolution of the two functions is equivalent to the Fourier transform of the product of the Fourier transforms of the functions.

We next recognize that the integration over $x^{\prime}$ in eq. (4) is equivalent to the Fourier transform of the function in $x^{\prime}$ evaluated at $\omega_{x^{\prime}}=0$. Realizing that this one dimensional Fourier transform in $x^{\prime}$ and the one-dimensional Fourier transform in $\nu$ in eq. (4) can be combined into a two-dimensional forward Fourier transform and reversing the direction of the axes $(r, \theta)$ in the output reconstruction space, we rewrite eq. (4) as

$\mu\left(r_{n}, \theta\right)=-\left.\frac{1}{2 \pi^{2}} \mathcal{F}\right|_{\omega_{x^{\prime}}=0}\left[F_{\phi}\left(x^{\prime}, \nu\right) G_{\phi}\left(r_{n}, x^{\prime}, \nu\right)\right]$.

We denote the variable $r$ by $r_{n}$ since the convolution over $\phi$ and the integration over $x^{\prime}$ in eq. (2) must be performed for a specific $r_{n}$ value. This is especially true in the optical implementation since the three variable function $g\left(r, x^{\prime}, \phi\right)$ cannot be realized in a twodimensional optical system. Instead, separate twodimensional functions $g\left(r_{n}, x^{\prime}, \phi\right)$ are needed for each $r_{n}$ value. The complete $\mu(r, \theta)$ reconstructed image is then formed from the individual $\mu\left(r_{n}, \theta\right)$ functions by $\Sigma_{r}$. The final form of the inverse Radon transform that we will optically implement is thus

$$
\begin{aligned}
& \mu(-r,-\theta)=-\left.\frac{1}{2 \pi^{2}} \sum_{n=1}^{\infty} \mathcal{F}\right|_{\omega_{x^{\prime}}=0}\left[F_{\phi}\left(x^{\prime}, \nu\right) G_{\phi}\left(r_{n}, x^{\prime}, \nu\right)\right. \\
& =-\left.\frac{1}{2 \pi^{2}} \sum_{n=1}^{\infty} \mathcal{F}\right|_{\omega_{x^{\prime}=0}}\left\{\mathcal{F}_{\phi}\left[f\left(x^{\prime}, \phi\right)\right]\right. \\
& \left.\quad \times \mathcal{F}_{\phi}\left[\frac{1}{\left(r_{n} \cos \phi-x^{\prime}\right)^{2}}\right]\right\} .
\end{aligned}
$$

\section{Optical implementation}

The optical system for realization of the reconstruction is shown in fig. 1. The input and filter plane functions are shown below the optical schematic. We first discuss the formation of the required complex filter functions $G_{\phi}$ at $\mathrm{P}_{1}$. These functions are most easily formed by calculating $\left[r \cos \phi-x^{\prime}\right]^{-2}$ by digital computer, plotting this function on a Calcomp system, subsequently photo reducing the pattern, and placing it at $\mathbf{P}_{0}$ (fig. 1). The complex Fourier transform of this pattern is then formed at $P_{1}$ by $L_{1}$ and the complex Fourier transform recordea by normal interference

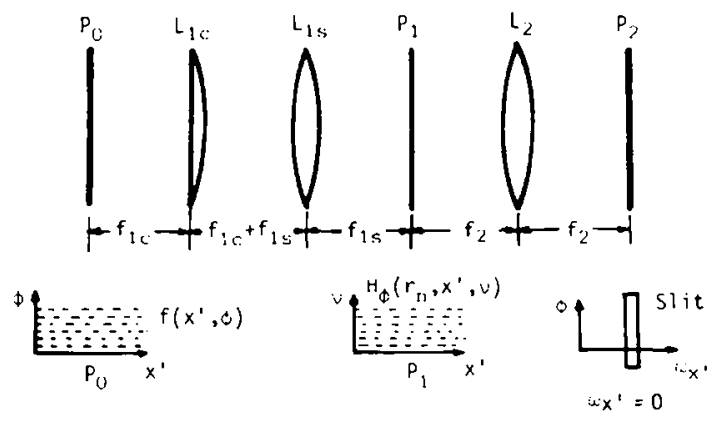

Fig. 1. Schematic diagram of an optical processor to realtze the inverse radon transform by convolution or correlation. 
methods [8]. The sampled $64 \times 64$ point input spatial domain reference mask $g\left(r_{n}, x^{\prime}, \phi\right)$ used is shown in fig. 2. For simplicity, the $x^{\prime}$ projection axis is normalized from +1 to -1 and a radially symmetric input object (fig. 3a) was chosen. This allows us to use only the projections from 0 to $\pi$ (as shown in the vertical axis of fig. 2).

With $f\left(x^{\prime}, \phi\right)$ recorded at $\mathrm{P}_{0}$ and $G_{\phi}\left(0.8, x^{\prime}, \nu\right)$ recorded holographically at $\mathrm{P}_{1}$, the output plane $\mathrm{P}_{2}$ pattern should consist of 4 bars (the equivalent polar coordinate version of fig. $3 \mathrm{a}$ ). This output reconstruction for the $G_{\phi}$ filter for $r=0.8$ is shown in fig. 3b. Only half of the output plane is shown and it is seen to consist of the proper slice (at $r=0.8$ ) of the $\mu(r, \theta)$ reconstruction. An isometric view of this output imagery construction slice is shown in fig. $3 c$. The length of the bars should theoretically be

$\frac{f_{\mathrm{Llc}}}{f_{\mathrm{Lls}}} \times \frac{f_{\mathrm{L} 2}}{f_{\mathrm{Llc}}} \times \frac{L}{4}=\frac{300}{381} \times \frac{190}{300} \times \frac{12.5}{4}=1.56 \mathrm{~mm}$,

where $L=12.5 \mathrm{~mm}$ is the input plane aperture used. The measured value of the slit width at the $50 \%$ points was $1.60 \mathrm{~mm}$ in excellent agreement with theory.

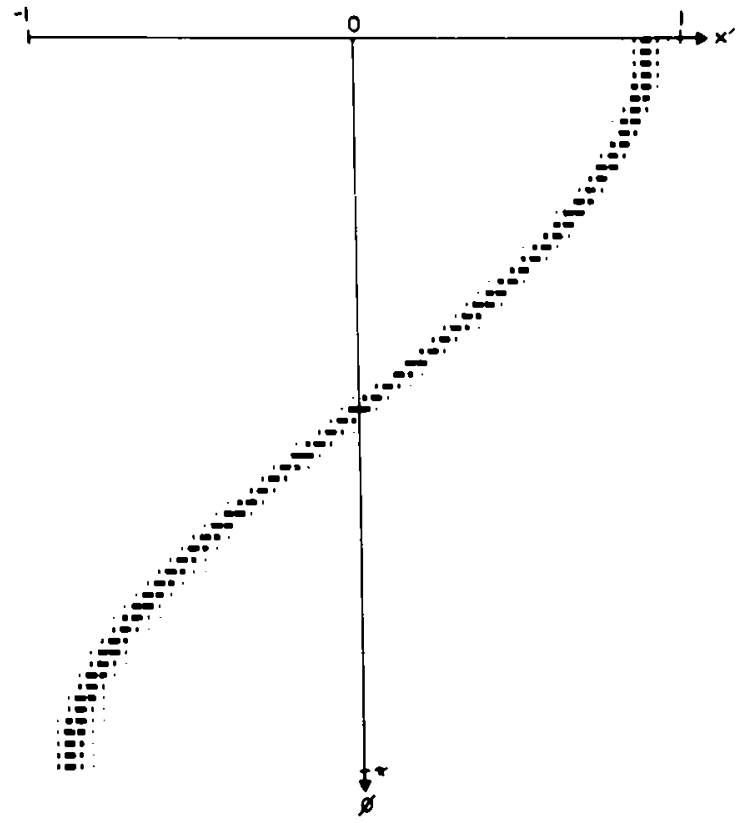

Fig. 2. $64 \times 64$ Input computer generated mask to realize the function $g\left(r_{n}, x^{\prime}, \phi\right)=\left[r_{n} \cos \phi-x^{\prime}\right]^{-2}$ from normalized values $x^{\prime}=-1$ to +1 and $\phi=0$ to $\pi$ for $r_{n}=0.8$.
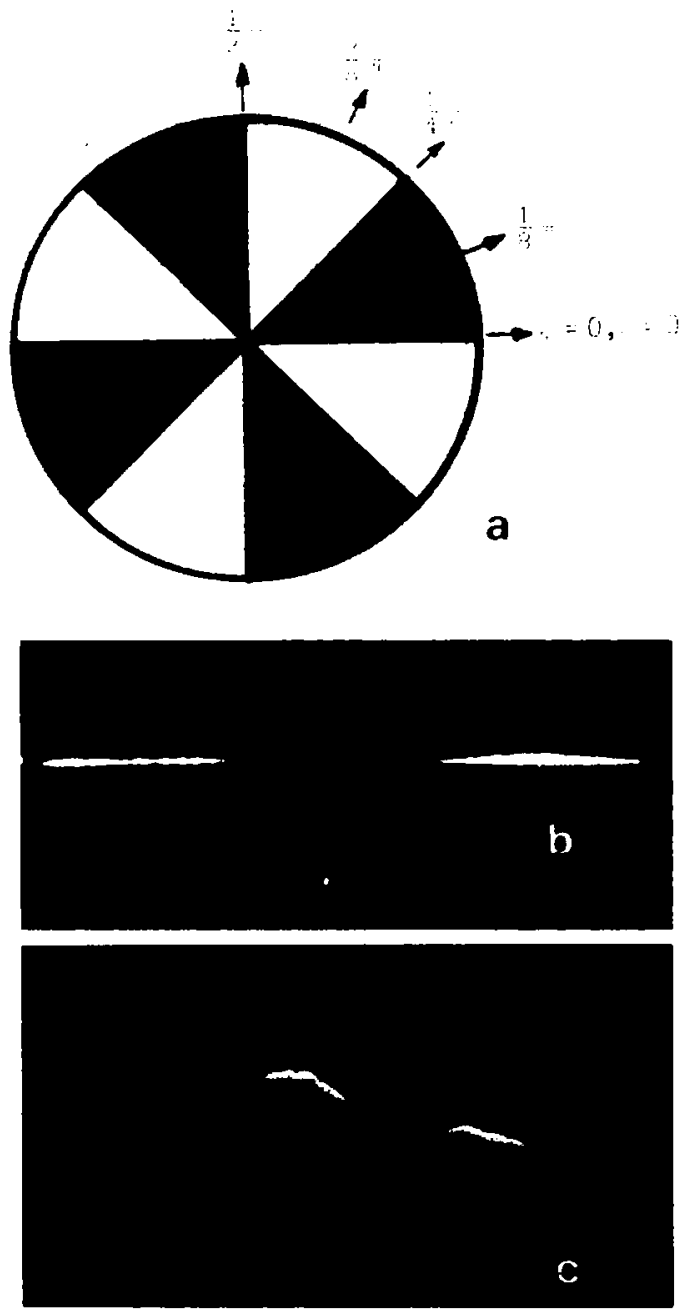

Fig. 3. (a) The input test object used. (b) Optical reconstruction using the inverse radon transform of the line through $r=0.8$ in the output $\mu(r, \theta)$. (c) Isometric display of the image in (b).

Although separate reference mask functions $G_{\phi}$ are required for each $r_{n}$, these reference masks are fixed once the recording geometry of the CT system and number of projections, etc. are fixed. The necessary reference masks can thus be permanently recorded on film which can then be easily and rapidly moved through plane $P_{1}$. To produce the reconstructed output image, the output plane $\mathrm{P}_{2}$ would be translated in synchronization with the film transport in $P_{1}$. 


\section{Acknowledgement}

The authors wish to thank Dr. D. Psaltis for initial technical discussions, T. Tullia, and T. Luu for laboratory assistance and the Office of Naval Research on contract NR366-005 for partial financial support of this research.

\section{References}

[1] J. Radon, Ber. Saechs. Akad. Wiss. (Leipzig) 69 (1917) 262.
[2] H.H. Barrett and W. Swindel, Proc. IEEE 65 (1977) 89.

[3] Opt. Engr., special issue on Computed Tomography, 16, No. 1 (1977).

[4] IEEE Trans. Nuc. Med., special issue on Physical and Computational Aspects of Three-Dimensional Imagery Construction, NS-21 (1974).

[5] C.M. Vest, J. Opt. Soc. Amer. 64 (1974) 1215.

[6] N.V. Berry and D.F. Gibbs, Proc. Royal Soc. (London) A314 (1970) 143.

[7] J.M. Gel'Fand, M.I. Graev and N.Ya. Vilenkin, Generalized functions, Vol. V (Academic Press, New York, 1966).

[8] M. Nishimuru, D. Casasent and F. Caimi, Opt. Commun. 24 (1978) 276.

[9] Vander Lugt, IEEE IT-10. 